HMG-CoA reductase inhibitor and lowers sterol precursor levels in Insig-DKO embryos. This experiment strongly supports the idea that elevated sterol precursors underlie the facial clefting. Future work will investigate whether this is mediated through an alteration of Shh signaling.

\section{Acknowledgments}

I would like to thank Steven Fliesler for his critical review of this manuscript. I also would like to express my gratitude to my patients and their parents, whose participation allows my group's research to proceed. This work was supported by the intramural program of the National Institute of Child Health and Human Development, NIH.

Address correspondence to: Forbes D. Porter, HDB, NICHD, NIH, Building 10, Room 9D42, 10 Center Dr., Bethesda, Maryland 20892, USA. Phone: (301) 435-4432; Fax: (301) 480-5791; E-mail: fdporter@ mail.nih.gov.
1. Kelley, R.I., and Hennekam, R.C. 2000. The SmithLemli-Opitz syndrome. J. Med. Genet. 37:321-335.

2. Irons, M., Elias, E.R., Salen, G., Tint, G.S., and Batta, A.K. 1993. Defective cholesterol biosynthesis in Smith-Lemli-Opitz syndrome. Lancet. 341:1414.

3. Porter, F.D. 2002. Malformation syndromes due to inborn errors of cholesterol synthesis. J. Clin. Invest. 110:715-724. doi:10.1172/JCI200216386.

4. Shackleton, C., Roitman, E., Guo, L.W., Wilson, W.K., and Porter, F.D. 2002. Identification of 7(8) and $8(9)$ unsaturated adrenal steroid metabolites produced by patients with 7-dehydrosterol-delta7reductase deficiency (Smith-Lemli-Opitz syndrome). J. Steroid Biochem. Mol. Biol. 82:225-232.

5. Wassif, C.A., Yu, J., Cui, J., Porter, F.D., and Javitt, N.B. 2003. 27-Hydroxylation of 7- and 8-dehydrocholesterol in Smith-Lemli-Opitz syndrome: a novel metabolic pathway. Steroids. 68:497-502.

6. Engelking, L.J., et al. 2006. Severe facial clefting in Insig-deficient mouse embryos caused by sterol accumulation and reversed by lovastatin. J. Clin. Invest. 116:2356-2365. doi:10.1172/JCI28988.

7. Goldstein, J.L., DeBose-Boyd, R.A., and Brown, M.S. 2006. Protein sensors for membrane sterols. Cell. 124:35-46.

8. Engelking, L.J., et al. 2005. Schoenheimer effect explained - feedback regulation of cholesterol synthesis in mice mediated by Insig proteins. J. Clin. Invest. 115:2489-2498. doi:10.1172/JCI25614.

9. Wassif, C.A., et al. 2001. Biochemical, phenotypic and neurophysiological characterization of a genetic mouse model of RSH/Smith-Lemli-Opitz syndrome. Hum. Mol. Genet. 10:555-564.

10. Krakowiak, P.A., et al. 2003. Lathosterolosis: an inborn error of human and murine cholesterol synthesis due to lathosterol 5-desaturase deficiency. Hum. Mol. Genet. 12:1631-1641.

11. Wechsler, A., et al. 2003. Generation of viable cholesterol-free mice. Science. 302:2087.

12. Porter, J.A., Young, K.E., and Beachy, P.A. 1996. Cholesterol modification of hedgehog signaling proteins in animal development. Science. 274:255-259.

13. Rice, R., et al. 2004. Disruption of Fgf10/Fgfr2bcoordinated epithelial-mesenchymal interactions causes cleft palate. J. Clin. Invest. 113:1692-1700. doi:10.1172/JCI200420384.

14. Lewis, P.M., et al. 2001. Cholesterol modification of sonic hedgehog is required for long-range signaling activity and effective modulation of signaling by Ptc1. Cell. 105:599-612.

15. Cooper, M.K., et al. 2003. A defective response to Hedgehog signaling in disorders of cholesterol biosynthesis. Nat. Genet. 33:508-513.

16. Corcoran, R.B., and Scott, M.P. 2006. Oxysterols stimulate Sonic hedgehog signal transduction and proliferation of medulloblastoma cells. Proc. Natl. Acad. Sci. U. S. A. 103:8408-8413.

17. Frank-Kamenetsky, M., et al. 2002. Small-molecule modulators of Hedgehog signaling: identification and characterization of Smoothened agonists and antagonists. J. Biol. 1:10.

\title{
Adaptive human regulatory T cells: myth or reality?
}

\section{Lucienne Chatenoud and Jean-François Bach}

Université René Descartes Paris 5, INSERM U580, Hôpital Necker-Enfants Malades, Paris, France.

\begin{abstract}
It is now well established that a distinct subset of $T$ lymphocytes is essential for downregulating immune responses to both endogenous (self) and exogenous antigens. These Tregs are $\mathrm{CD}^{+}$and express high levels of CD25 (the $\alpha$ chain of the IL-2 receptor) and the transcription factor Foxp3. The mechanisms determining the lifespan, homeostasis, and in vivo generation of these Tregs are still ill defined. A study by Vukmanovic-Stejic et al. in this issue of the JCI shows that in humans, Tregs are present throughout life but that despite their high throughput, they are short lived (see the related article beginning on page 2423 ). It is thus unlikely that all CD4 ${ }^{+} \mathrm{CD} 25^{\mathrm{hi}} \mathrm{Foxp} 3^{+}$ Tregs are generated as a separate lineage in the thymus. The authors propose that during adulthood, Tregs essentially emerge at the periphery from the memory $\mathrm{T}$ cell pool.
\end{abstract}

The existence in humans of naturally occurring $\mathrm{CD} 4^{+} \mathrm{CD} 25^{+}$Tregs is highlighted by the rapid onset of a severe polyautoimmune syndrome in children genetically deficient for the transcription factor Foxp3, a lineage marker for this Treg subset. Based on this observation in humans and also on

Conflict of interest: The authors have declared that no conflict of interest exists.

Citation for this article: J. Clin. Invest. 116:2325-2327 (2006). doi:10.1172/JCI29748. compelling experimental evidence obtained from the study of mice (1-4), it is postulated and generally accepted that Tregs are responsible for the control of autoreactive $\mathrm{T}$ cells, which react aggressively toward the host's tissues and trigger autoimmune disease in the absence of effective $T$ cell-mediated regulation.

A growing number of studies indicate that Tregs also play a significant role in downregulating the immune response to a variety of nonself antigens, such as micro- bial antigens, tumor antigens, and alloantigens (5-7). Some confusion persists, however, concerning the precise definition of the cell phenotype, functional capacity, and fine antigen specificity of the Tregs participating in these diverse contexts. The situation is further complicated by the fact that some studies argue for the participation of selective and distinct types of Tregs under each of these different conditions (8-13). In contrast, other studies adopt a more unifying view and propose that a single population of $\mathrm{T}$ cells, namely, thymus-derived $\mathrm{CD} 4{ }^{+} \mathrm{CD} 25^{\text {hi }} \mathrm{Foxp}^{+} \mathrm{T}$ cells, gives rise to Tregs controlling the various immune responses (5).

It is apparent that these latter $\mathrm{CD}_{4}^{+}$ $\mathrm{CD} 25^{\text {hiFoxp }} 3^{+} \mathrm{T}$ cells have a predominant role in controlling autoimmune responses in normal individuals in a phenomenon known as physiological tolerance. It is probably an oversimplification, however, to infer that these cells play a role in the control of all other immune responses, especially since effective immune regulation is 


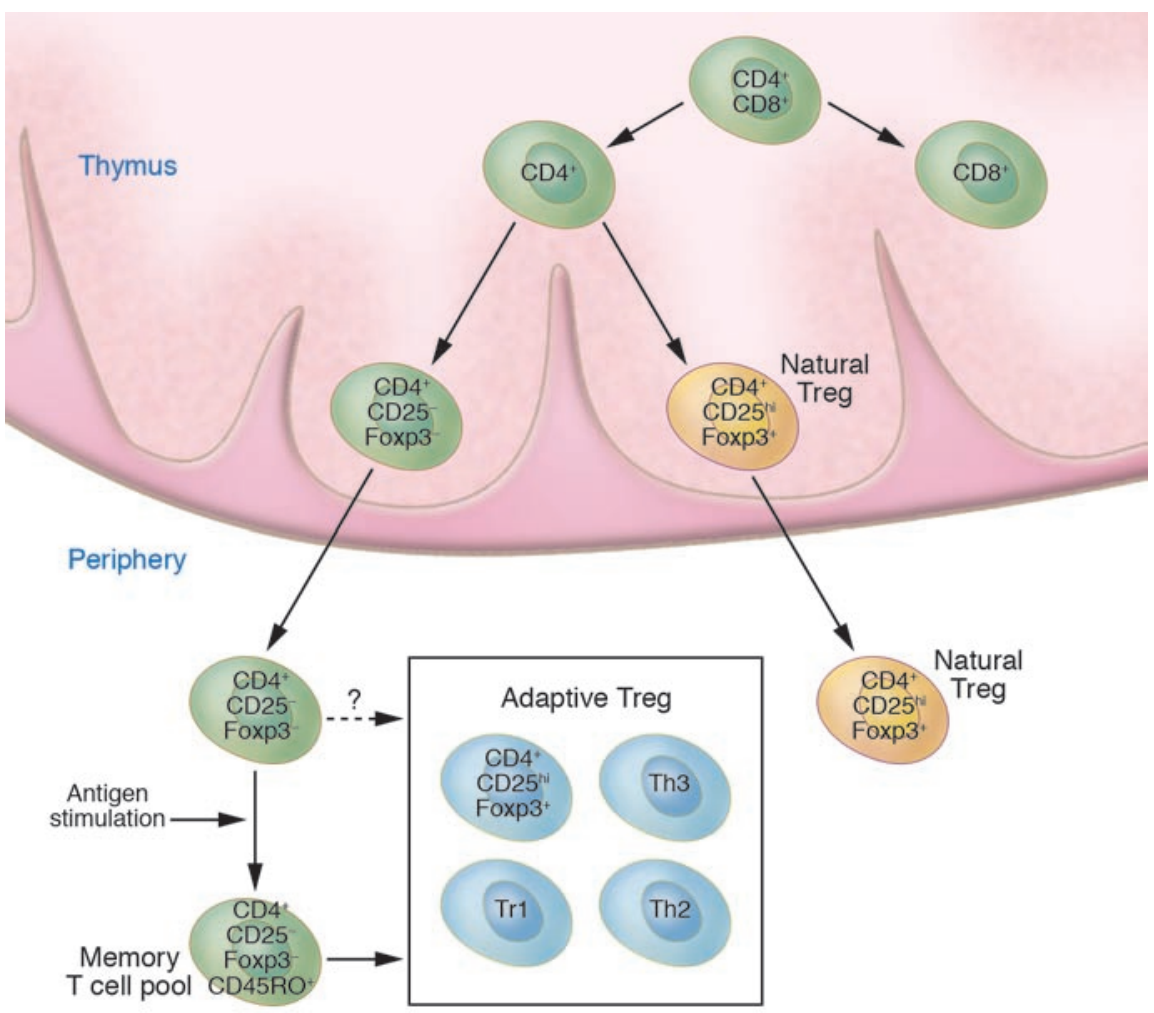

Figure 1

T lymphocytes originate from the thymus. Evidence has been accumulated to demonstrate that "natural" Tregs, whose major function is to control autoimmune responses, stem as a separate lineage in the thymus. These natural Tregs are $\mathrm{CD}^{+}$, they express the transcription factor Foxp3, which represents a lineage marker, and high levels of CD25. Natural Tregs that migrate to the periphery keep their phenotypic and functional properties, which are essentially cytokine independent. In parallel, other subsets of CD4+ Tregs have been described that very effectively control immune responses not only to self antigens but also to a wide variety of nonself antigens (microbial, tumoral, and transplantation antigens). These Tregs are not present as such in the thymus; they derive from peripheral precursors that are CD4+CD25- and differentiate into functional Tregs following adequate stimulation (in the presence of the cognate antigen and specialized immunoregulatory cytokines, i.e., TGF- $\beta$, IL-10, and IL-4). They are generally termed "adaptive" Tregs. Once differentiated, adaptive Tregs, like natural Tregs, may express CD25 and Foxp3. However, one main feature that differentiates adaptive from natural Tregs is their unique cytokine dependence. The study by Vukmanovic-Stejic et al. in this issue of the $\mathrm{JCl}(14)$ proposes that, in humans, even at a very advanced age, adaptive Tregs essentially emerge from CD4+CD25- $\mathrm{T}$ cells belonging to the memory $\mathrm{T}$ cell pool (previously primed by cognate antigens). Presently, one cannot exclude, however, that especially in young adults, some adaptive Tregs may emerge from naive, peripheral CD4+CD25- T cells. Tr1, Treg type 1.

expected to last a lifetime. Because of thymic involution at the time of puberty, one would then have to postulate that all Tregs constitute a pool of self-perpetuating, longterm-surviving thymic emigrants. This appears unlikely, especially in humans.

\section{Characteristics of adaptive Tregs in humans}

It is this complex, though highly relevant, problem that Akbar's group addresses in their study in this issue of the JCI (14). Akbar, Vukmanovic-Stejic, and colleagues present data showing that the proportion and functional integrity of $\mathrm{CD} 4{ }^{+} \mathrm{CD} 25^{\text {hi }} \mathrm{Foxp}^{+}$Tregs are maintained in older ( $>70$ years) human subjects and that these cells most probably do not derive from the thymic lineage of $\mathrm{CD}^{+} \mathrm{CD} 25^{\text {hiFoxp }} 3^{+}$Tregs.

The authors used an original method based on the in vivo incorporation of deuterium-labeled glucose (or glucose derivatives) into the DNA of dividing cells, with ultimate follow-up of the labeled population and subsequent evaluation of cell replication and survival within phenotypically distinct peripheral $\mathrm{T}$ cell subsets (14).
The results obtained convincingly show that throughout the individual's whole life span, lymphocytes corresponding to the phenotypic and in vitro functional definition of Tregs are generated from the peripheral pool of $\mathrm{CD}^{+} \mathrm{CD} 45 \mathrm{RO}^{+} \mathrm{CD} 25^{-}$ Foxp $3^{-}$memory T cells (Figure 1).

The distinction between "natural" Tregs of thymic origin and "adaptive" Tregs exclusively generated from peripheral CD25- lymphocytes following adequate conditions (including antigen stimulation and cytokine milieu) was suggested a few years ago from data obtained and validated in the mouse. It was then shown that peripheral CD25- $\mathrm{T}$ cells whose $\mathrm{T}$ cell receptor or coreceptors (e.g., CD4) are stimulated in the presence of TGF- $\beta$ acquire regulatory properties that may be assessed both in vitro and in vivo $(2,4,15,16)$.

The results reported here by Vukmanovic-Stejic et al. (14) may represent the first indication of a similar dichotomy in human Tregs. If confirmed, 2 other sets of data are of particular interest, namely those showing that human Tregs have a shorter doubling time when compared with other subsets studied (e.g., peripheral classical memory and naive-type $\mathrm{T}$ cells) and that they appear particularly sensitive to apoptosis. Such observations may present a solid argument for the dependence of human adaptive Tregs on continuous antigen stimulation and/or the presence of growth factors in their immediate environment for their differentiation and survival. This could turn out to be an essential condition of their homeostasis.

\section{Pending issues}

These conclusions should be tempered, however, by an appreciation of the technical limitations of investigations performed in humans. The first concern is that the rationale of the work and its interpretations are based on the assumption that a high level of CD25 expression is a reliable marker for human Tregs. It is unquestionable that the $\mathrm{CD} 4^{+} \mathrm{CD} 25^{\text {hi }} \mathrm{T}$ cell subset concentrates both the majority of Foxp $3^{+}$ cells and the essential part of functional regulatory capacity as assessed by in vitro coculture. However, how sure are we that these in vitro functional assays in humans reflect an in vivo regulatory functional capacity as shown in the mouse, using adoptive transfer experiments? Moreover, recent data suggest that the IL-7 receptor (CD127) is downregulated on a subset of human peripheral $\mathrm{CD} 4^{+} \mathrm{T}$ cells that are 
Foxp $3^{+}$and suppressive but that express no or low levels of CD25 (17). Additionally, one may consider whether Foxp3 in the human as opposed to the mouse is also expressed by activated $T$ cells, independently of any regulatory function.

The second concern, closely linked to the first, is the critical issue of the antigen specificity of the Tregs studied, which is only very indirectly addressed (14). The results showing a biased $\mathrm{T}$ cell repertoire restricted to a given $V \beta$ family ( $V \beta 2$ in this case) in a representative individual with persistent CMV infection are intriguing. However, the regulatory functional capacity of the $\mathrm{CD}^{+} \mathrm{CD} 25^{\text {hiFoxp }}{ }^{+}$cell subset detected in this $\mathrm{V} \beta 2$ anti-CMV population remains to be demonstrated.

To conclude, it appears plausible to extend to the human the dichotomy proposed in the mouse that distinguishes natural versus adaptive Tregs, which have distinct origins, namely, thymic-derived $\mathrm{CD} 4^{+} \mathrm{CD} 25^{+} \mathrm{Foxp}^{+}$cells in the case of natural regulatory lymphocytes versus peripheral memory-type $\mathrm{CD} 4^{+} \mathrm{CD} 25^{-}$precursors in the case of adaptive Tregs. In this context, it will be important to further experimentally dissect the adaptive Treg subset to more directly address whether or not the differences that have been proposed for each subset (e.g., Th2, Th3, Tr1, CD45RB low $\mathrm{T}$ cells) in terms of phenotype and putative cytokine dependency are indeed a reflection of their belonging to distinct cell lineages.

Address correspondence to: Jean-François Bach, INSERM U580, Hôpital NeckerEnfants Malades, 161 Rue de Sèvres, 75015 Paris, France. Phone: 33-144-49-53-73; Fax: 33-143-06-23-88; E-mail: bach@necker.fr.

1. Hori, S., Takahashi, T., and Sakaguchi, S. 2003. Control of autoimmunity by naturally arising regulatory CD4+ T cells. Adv. Immunol. 81:331-371.

2. Bach, J.-F. 2003. Regulatory T cells under scrutiny. Nat. Rev. Immunol. 3:189-198.

3. Chatenoud, L., Salomon, B., and Bluestone, J.A. 2001. Suppressor T cells-they're back and critical for regulation of autoimmunity! Immunol. Rev. 182:149-163

4. Bluestone, J.A., and Abbas, A.K. 2003. Natural versus adaptive regulatory T cells. Nat. Rev. Immunol. 3:253-257.

5. Sakaguchi, S. 2005. Naturally arising Foxp3expressing $\mathrm{CD} 25+\mathrm{CD} 4+$ regulatory $\mathrm{T}$ cells in immunological tolerance to self and non-self. Nat. Immunol. 6:345-352.

6. Waldmann, H., et al. 2006. Regulatory T cells in transplantation. Semin. Immunol. 18:111-119.

7. Coombes, J.L., Robinson, N.J., Maloy, K.J., Uhlig, H.H., and Powrie, F. 2005. Regulatory T cells and intestinal homeostasis. Immunol. Rev. 204:184-194. 8. Groux, H., et al. 1997. A CD4+ T-cell subset inhibits antigen-specific T-cell responses and prevents colitis. Nature. 389:737-742.

9. Roncarolo, M.G., and Levings, M.K. 2000. The role of different subsets of T regulatory cells in controlling autoimmunity. Curr. Opin. Immunol. 12:676-683.

10. Levings, M.K., et al. 2002. Human CD25+CD4+ $\mathrm{T}$ suppressor cell clones produce transforming growth factor beta, but not interleukin 10 , and are distinct from type $1 \mathrm{~T}$ regulatory cells. J. Exp. Med. 196:1335-1346.

11. Weiner, H.L., et al. 1994. Oral tolerance: immunologic mechanisms and treatment of animal and human organ-specific autoimmune diseases by oral administration of autoantigens. Annu. Rev. Immunol. 12:809-837.

12. Ochi, H., et al. 2006. Oral CD3-specific antibody suppresses autoimmune encephalomyelitis by inducing CD4(+)CD25(-)LAP(+) T cells. Nat. Med. 12:627-635.

13. Tisch, R., Wang, B., Atkinson, M.A., Serreze, D.V., and Friedline, R. 2001. A glutamic acid decarboxylase 65 -specific Th2 cell clone immunoregulates autoimmune diabetes in nonobese diabetic mice. J. Immunol. 166:6925-6936.

14. Vukmanovic-Stejic, M., et al. 2006. Human $\mathrm{CD} 4^{+} \mathrm{CD} 25^{\text {hi }} \mathrm{Foxp}^{+}$regulatory $\mathrm{T}$ cells are derived by rapid turnover of memory populations in vivo. J. Clin. Invest. 116:2423-2433. doi:10.1172/JCI28941.

15. Chen, W., et al. 2003. Conversion of peripheral CD4+CD25- naive $\mathrm{T}$ cells to $\mathrm{CD} 4+\mathrm{CD} 25+$ regulatory $\mathrm{T}$ cells by TGF-beta induction of transcription factor Foxp3. J. Exp. Med. 198:1875-1886.

16. Cobbold, S.P., et al. 2004. Induction of foxP3+ regulatory $\mathrm{T}$ cells in the periphery of $\mathrm{T}$ cell receptor transgenic mice tolerized to transplants. J. Immunol. 172:6003-6010.

17. Liu, W., et al. 2006. CD127 expression inversely correlates with FoxP3 and suppressive function of human CD4(+) T reg cells. J. Exp. Med. 203:1701-1711.

\title{
You say estren, I say estrogen. Let's call the whole replacement off!
}

\author{
Ushma S. Neill
}

Journal of Clinical Investigation, Columbia University College of Physicians and Surgeons, New York, New York, USA.

\begin{abstract}
Estrogens and androgens play a key role in regulating bone mass. However, their clinical use as bone anabolic agents is limited due to unwanted side effects, particularly in reproductive organs. In 2002, the synthetic ligand estren was described to reproduce the bone anabolic, nongenotropic effects of sex steroids while having no effect on the uterus or seminal vesicles. But in the current issue of the JCI, Windahl et al. provide data showing that estrens are not as suitable a replacement for estrogen as was initially reported (see the related article beginning on page 2500). Though not catabolic, estrens triggered only minor, nonsignificant increases in bone mass in gonadectomized mice, all the while inducing hypertrophy of reproductive organs. Does this mean estrens should not be pursued as a therapy for osteoporosis?
\end{abstract}

Nonstandard abbreviations used: AR, androgen receptor; ER, estrogen receptor; SERM, selective estrogen receptor modulator.

Conflict of interest: The author has declared that no conflict of interest exists.

Citation for this article: J. Clin. Invest. 116:2327-2329 (2006). doi:10.1172/JCI29733.

\section{Estrogen and its receptors}

The estrogen hormone family plays an essential role in the regulation of skeletal growth and homeostasis. While osteoblasts, osteocytes, and osteoclasts can be indirect targets of hormone signaling, they are also direct targets of estrogen and express functional estrogen and androgen receptors (ER and AR, respectively) (1). As estrogen or androgen deficiency can lead to rapid decreases in bone mass, therapies designed to return these sex hormones to their original levels would seem logical. However, these strategies have been fraught with difficulty due to the complex nature of hormone signaling.

In the classical (genomic) model of estrogen signaling, estrogens bind to the ER in the nucleus (Figure 1). Over the course of several hours, the estrogen-ER complex then induces a direct response through estrogen response element sequences or an indirect response by triggering expression of other proteins such as transcription factors of the AP1 family, among others. This is viewed as the main 